\title{
The Effects of Endometrial Scratch on Pregnancy Rate in IUI Cycles
}

\author{
Kobra Hamdi ${ }^{\circledR}$, Nafiseh Moayyed Nia $^{2}$, Parvin Hakimi ${ }^{*}$, Alieh Ghasemzadeh ${ }^{1}$
}

\begin{abstract}
Objectives: Infertility is considered as a major issue all over the world. All the studies in this field focus on how to develop successful methods of turning infertile couples into fertile ones. Endometrial mechanical injury in order to augment the probability of implantation of embryo and pregnancy rate has been particularly noted in recent years. Considering the controversies in the use of methods and results of the previous studies, we decided to compare the pregnancy and abortion rates in the intrauterine insemination (IUI) cycles with and without intervention, by employing a new method.

Materials and Methods: This interventional study was performed on 150 infertile couples who referred to the infertility treatment clinic of Al-Zahra hospital, Tabriz (from April 2016 to March 2017) and were randomly divided into two groups. The IUI procedure was carried out in the first group after the endometrial scratch on the first to fifth days of the menstrual cycle, while the control group underwent only IUI without any intervention. The pregnancy and abortion rates were compared in study groups.

Results: Pregnancy rate in the intervention group was significantly higher compared to the control group. No relationship was observed between the abortion rate and the intervention. There was no statistically significant difference between type and cause of infertility, maternal age and body mass index (BMI), duration of infertility, number of dominant follicles and pregnancy or abortion rates.

Conclusions: The endometrial scratching performed on the first to fifth days of the menstrual cycle, preceding IUI procedure, leads to a significant increase in pregnancy rate. On the other hand, this method can be employed with lower costs in comparison to other fertilization methods.

Keywords: Infertility, Pregnancy, Endometrial scratch, IUI
\end{abstract}

\section{Introduction}

Infertility poses a major problem, whereas $10-15 \%$ of couples have been reported to be facing this issue (1, $2)$. The considerations for evaluation and treatment of infertility usually start after a year of unsuccessful pregnancy in couples without contraceptive methods. However, in women over 35 years of age, this period should be considered 6 months (3). According to numerous studies, infertile couples without any treatment have $2 \%$ to $4 \%$ chance of pregnancy in a monthly cycle, while normal couples have $20 \%-25 \%$ chance of pregnancy in a monthly cycle, showing $80 \%-90 \%$ less chance of pregnancy. As the duration of infertility increases in young couples, the necessity for intervention rises $(3,4)$. The most widelyused methods like intrauterine insemination (IUI), ovulation induction with oral or injectable medications, combination of IUI and ovulation induction, and assisted reproductive technologies (ARTs) are proposed empirical regimens (4).

Among the causes of implantation failure, uterine problems have gained more attention in recent years. Some of these issues are as follows: thin endometrium, altered expression of adhesion molecules and immunological factors leading to weak endometrial receptivity, and genetic abnormalities in males or females (5).

It has been stated that in an inflammatory condition, the chance of implantation decreases, and in recent years the scratching of the endometrium, defined as an intentional damage to the endometrial lining using a biopsy or curettage to improve endometrial receptivity, has been proposed to play a role in increasing the chances of implantation (6). However, studies show different and in some cases paradoxical results, depending on the day of performing the scratching. This study was designed to evaluate the effects of scratching from the first to the fifth day of the menstrual cycle, prior to IUI, and compare the pregnancy and abortion rates in cycles with and without the intervention.

\section{Materials and Methods}

Among the infertile couples referred to infertility treatment clinic of Al-Zahra hospital (from April 2016 to March 2017), 150 cases were chosen randomly to enter this randomized clinical trial.

Only couples who were candidates for IUI with the following inclusion criteria entered the study: Mild 
ovulation disorder, mildly abnormal semen parameters like sperm counts $\geq 15$ million/mL, sperm motility higher than $20 \%$, and normal sperm morphology greater than $15 \%$, mild endometriosis, and infertility with an unknown etiology.

On the other hand, exclusion criteria included age over 35 , uterine masses like submucosal leiomyoma, previous diagnosis of moderate to severe pelvic endometriosis in abdominal or pelvic sonography, hysteroscopy or laparoscopy, unilateral obliteration of fallopian tube, females with the BMI (body mass index) over $35 \mathrm{~kg} / \mathrm{m}^{2}$, and severe abnormalities in seminal fluid.

More than 200 couples who referred to the Al Zahra hospital were eligible to enter the study, however, 150 of them entered the study based on randomization. Seminal fluid samples were collected in sterile plastic containers after 2 to 7 days of abstinence. Then, the analysis of these fluid samples was carried out based on WHO criteria. The analysis included sperm count, motility and viability.

The patients who met the inclusion criteria were randomly divided into two groups. Group one named as the 'case group' underwent IUI with endometrial scratch using an IUI catheter or pipelle biopsy, between the first and fifth day of menstrual cycle, while sonography was performed simultaneously, while group two, the 'control group', underwent IUI without scratching of the endometrium.

To perform endometrial scratching, the cervix was exposed using a sterile speculum and then the uterine entrance got cleansed using sterile gauze and saline. Then, the lining of the anterior and posterior wall of the uterus was gently scratched by inserting and moving a thin catheter up and down.

Ovulation stimulation was done similarly in both groups using $100 \mathrm{mg}$ clomiphene tablets, beginning from the third up to the fifth days of the menstrual cycle for 5 days. In addition, FSH (Gonal F, 75 units) was injected for 3 to 5 days, between the seventh and tenth days of the menstrual cycle.

After 10 to 12 days, the patients were visited and sonography was performed in order to evaluate the growth of the follicles and in cases with insufficient growth, more FSH was administered. For the follicles which were big enough (18-20 mm), HCG (5000-10 000 units) was administered intramuscularly, and after 36 hours, IUI was performed.

Moreover, luteal phase was supported by daily administration of $10 \mathrm{mg}$ dydrogesterone (Duphaston) for 14 days. Successful pregnancy after IUI was evaluated by beta human chorionic gonadotropin ( $\beta$-hCG) titers and sonography. The patients were followed-up for 3 months to assess the possibility of abortion.

\section{Statistical Analysis}

The obtained results were analyzed using SPSS software version 17.0. In order to compare the differences between groups, ANOVA and independent $t$ test were used, while chi-square test was used for analyzing nominal data. A $P$ value less than 0.05 was considered statistically significant.

\section{Results}

A total of 150 couples were included in this study and they were divided into two groups of 75 patients. The Mean age of the patients in the case group was $28.48 \pm 4.05$ years, while it was $28.05 \pm 4.77$ in the control group. The mean $\mathrm{BMI}$ of the patients was $26.81 \pm 4.37$ in the case group and it was $27.31 \pm 4.32$ in the control group. The infertility duration for couples in the case group was 4 years and 11 months with the standard deviation (SD) of 2 years and 8 months, whereas for the control group it was 5 years and 2 months with SD of 2 years and 10 months (Table $1)$. Among the study population, 102 couples $(68 \%)$ had primary infertility and 48 couples (32\%) had secondary infertility. In the case group, 54 couples $(72 \%$ of the couples in case group) and in the control group 48 couples (64\%) had primary infertility, while secondary infertility was observed in 21 couples (28\%) of the case group and 27 couples (36\%) of the control group (Table 2). No statistically significant difference was observed between two groups in terms of the types of infertility $(P=0.29)$.

The etiology of infertility in this study included (Figure 1): 1 . mild male infertility issues which were observed in 25 couples (17\%), 13 couples (17\%) in the case group and 12 couples (16\%) in the control group had this issue, 2. ovulation disorders which were observed in 57 couples (38\%), 28 couples (37\%) in the case group and 29 couples $(38 \%)$ in the control group had the same disorder, and 3. unknown etiology which was observed in 67 couples (37\%), 33 couples (44\%) in case group and 34 couples (45\%) in the control group. Only one couple in case group had mild endometriosis, which was statistically negligible and consequently, no statistically significant difference was observed between the case and control groups, regarding the etiology of infertility $(P=0.97)$. Among 150 couples

Table 1. The General Characteristics of the Patients in Case and Control Groups

\begin{tabular}{llll}
\hline $\begin{array}{l}\text { General } \\
\text { Characteristics }\end{array}$ & $\begin{array}{l}\text { Age }(\mathbf{y}) \\
(\text { mean } \pm S D)\end{array}$ & $\begin{array}{l}\text { BMI }\left(\mathrm{kg} / \mathrm{m}^{2}\right) \\
(\text { mean } \pm S D)\end{array}$ & $\begin{array}{l}\text { Duration of } \\
\text { Infertility (mean) }\end{array}$ \\
\hline Case group & $28.48 \pm 4.05$ & $26.81 \pm 4.37$ & $\begin{array}{l}4 \text { years and } 11 \\
\text { months }\end{array}$ \\
Control group & $28.05 \pm 4.77$ & $27.31 \pm 4.32$ & $\begin{array}{l}5 \text { years and 2 } \\
\text { months }\end{array}$ \\
\hline
\end{tabular}

Table 2. Type of Infertility in Case and Control Groups

\begin{tabular}{lcc}
\hline Type of infertility & Primary & Secondary \\
\hline Overall & 102 couples (68\%) & 48 couples (32\%) \\
Case group & 54 couples & 21 couples \\
Control Group & 48 couples & 27 couples \\
\hline
\end{tabular}


who entered this study, 21 couples (14\%) got pregnant successfully, 15 of them were in the case group and 6 were in the control group (Figure 2). Therefore, $71 \%$ of the pregnant cases in this study were from the case group, showing that two-thirds of the success in treating infertile couples in this study belonged to scratching method. Statistical analysis showed a significant relationship between implementing scratching method and increased pregnancy rates $(P=0.034)$. Among these 21 couples that got pregnant successfully, 14 couples $(66 \%)$ had primary infertility issues, while 7 couples (33\%) had secondary infertility disorders. However, there was no significant difference between two groups in terms of pregnancy rate and infertility type $(P=0.88)$. On the other hand, among these couples, 3 of them (14\%) had mild male infertility issues, 13 couples $(61 \%)$ had ovulatory disorders and 5 couples (24\%) had unknown etiology. No statistically significant difference was observed between groups in this regard $(P=0.09)$.

Most of the pregnancies happened at the age of 29 and had a significant relation with samples age distribution
$(P=0.013)$. Indeed, most of the patients were in the age range of 24-32, and obviously their higher rate of pregnancy was predictable. Nonetheless, the relationship between age and pregnancy was not statistically significant in the groups $(P=0.21)$.

Six cases (30\%) had normal BMI (18.5-24.9), 10 cases (47\%) were overweight (25-29.9) and 5 of them (23\%) were obese (30-34.9). No significant difference was observed in this regard $(P>0.05)$.

In addition, sonographic results demonstrated that 67 cases (44\%) had one dominant follicle and 11 cases (52\%) with successful fertilization had the same condition. On the other hand, 4 pregnant women (19\%) had 2 and 4 others had 3 dominant follicles. Only 2 of them (9\%) had 4 dominant follicles. However, in none of those groups, a significant relationship was observed between the number of dominant follicles and pregnancy success $(P=0.22)$.

The mean endometrial thickness was $8.39 \pm 1.06 \mathrm{~mm}$ in case group and $8.32 \pm 1.30 \mathrm{~mm}$ in the control group. In case group, the mean endometrial thickness in subjects that got pregnant successfully was $8.33 \pm 0.97$, while in the control

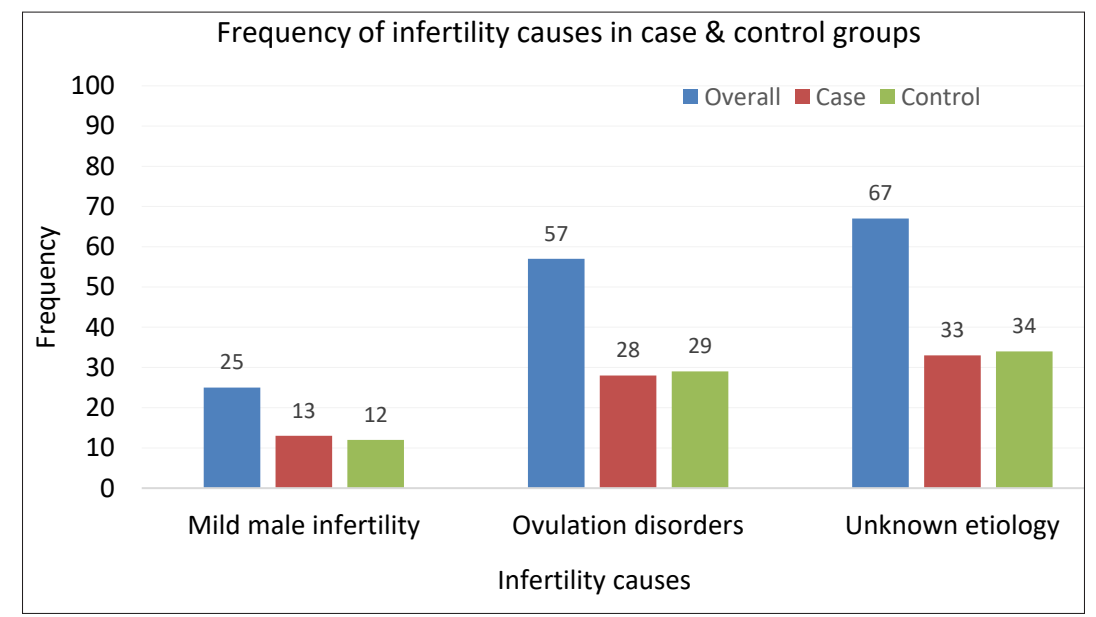

Figure 1. The Frequency of Infertility Causes in Case and Control Groups.

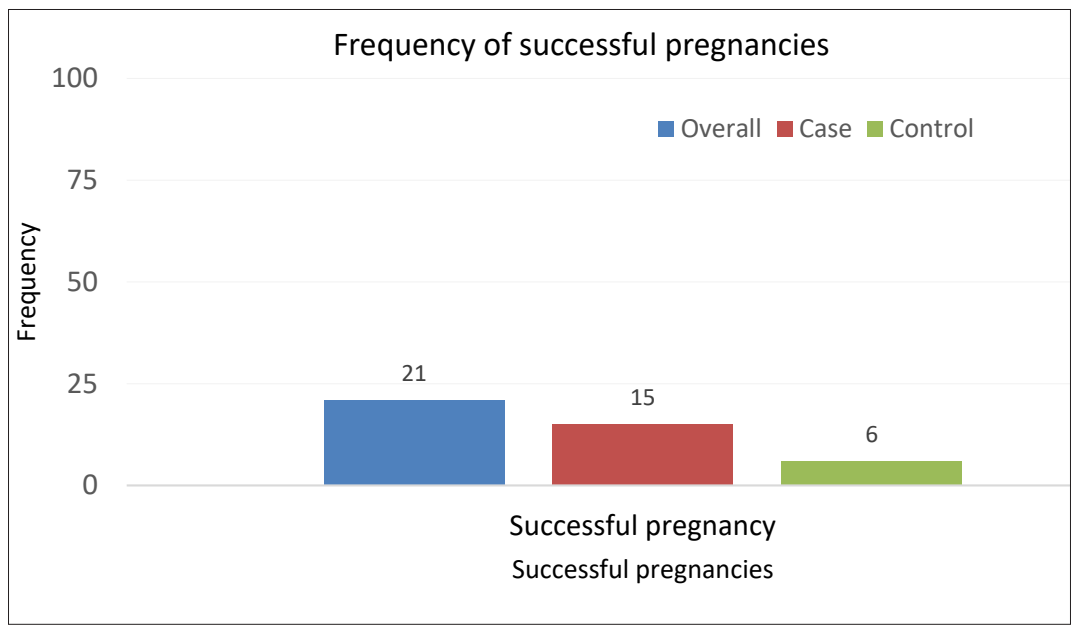

Figure 2. The Frequency of Successful Pregnancies in Case and Control Groups. 
group, it was $10.5 \pm 1.37$. There was also a significant relationship between endometrium thickness and pregnancy between groups, showing a higher endometrial thickness in the control group $(P=0.001)$.

Nonetheless, among these successful pregnancies, 6 cases of abortion were reported, showing a failure rate of $28 \%$, which included 3 couples from case $(20 \%$ of these couples) and 3 from the control group (50\% of this group) (Table 3). However, there was no significant difference between the groups, which might be due to the small size of samples $(P=0.16)$. On the other hand, there was a significant relationship between endometrial thickness and abortion, showing that the thicker the endometrium, the higher the rates of abortion $(P<0.05)$, though due to the lower number of abortions, this might not be reliable.

\section{Discussion}

Infertility is a serious issue for couples, and despite the advancement of technology, assisted reproduction methods fail to provide successful services with reasonable cost, fewer side-effects, and lower abortion rates.

This study aimed at evaluating the effects of endometrial scratch on pregnancy rates in IUI cycles. The results demonstrated a higher rate of pregnancy in the case group with a significant statistical difference in comparison to the control group. Similar studies in this regard have shown different results. Some were consistent with our study (79), while others showed that there are no relationships between endometrial injury and pregnancy rate (10). On the contrary, there were studies that reported a negative effect of endometrial injury on implantation (11).

Previous studies have reported different success rates for IUI. This method with clomiphene citrate has shown a pregnancy rate of $7 \%$ (12), while IUI with FSH (follicle stimulating hormone) showed an efficacy of $12 \%$ (13). On the other hand, other studies have reported a pregnancy rate of $18 \%$ for IUI in couples with unknown causes of infertility. The pregnancy rate in our study was $21 \%$, while in a study conducted by Maged et al (14), it was $28 \%$. However, our results showed an acceptably higher pregnancy rate in comparison to methods that implemented only IUI.

However, the time for inducing an injury seems to be controversial. For instance, in a study by Karimzade et al (11), a negative impact of local damage to the endometrium in the process of implantation in an IVF cycle, in the days that oocytes are available, was reported. On the other hand, Zarei et al in 2014 (10) indicated that local endometrium injury before IUI is not accompanied by increased pregnancy rate, decreased abortion and increased endometrial thickness. Therefore, the optimal days for the scratching was evaluated in this study and the first to fifth days of the menstrual cycle in the same IUI cycle showed positive results.

Besides, in a study by Abdelhamid (15), similar results
Table 3. The Frequency and Percentage of Aborted Cases among Successful Pregnancies in Case and Control Groups

\begin{tabular}{lcc}
\hline Type of infertility & Primary & Secondary \\
\hline Overall & 102 couples (68\%) & 48 couples (32\%) \\
Case group & 54 couples & 21 couples \\
Control Group & 48 couples & 27 couples \\
\hline
\end{tabular}

were obtained, reporting that the scratching method is better to be implemented before ovulation, which was performed on the eighth and ninth days of the menstrual cycle. Maged et al in 2016 (14) showed that promising results are obtained when the endometrial scratch is carried out one month prior to IUI in infertile patients with unknown etiology. This finding was consistent with our study, showing a high pregnancy rate using both scratching method and IUI, while, unlike our study, it showed that the efficient time to perform scratching is a cycle earlier. On the other hand, this study highlighted the low costs of scratching method, which might be a motivation for couples to undergo this method repeatedly.

Due to the small size of samples, most of the patients were in the age range of 25-30 and also had a BMI of around 25-30. Therefore, though most of the pregnancies and abortions happened in these age and BMI ranges, it won't be true if one deduces that these are the desirable characteristics for this method to work.

Moreover, no results were found in the literature regarding the abortion following the scratching method and IUI. However, differences in the results of the groups of this study might be due to the lower number of positive results. Finally, more studies with larger sample size and different etiologies of infertility are required in order to develop a better treatment plan with lower expenses for patients.

\section{Conclusions}

Most of the methods used for fertilization are too expensive considering their high rates of failure. However, in this study, using endometrium scratch method, the rate of pregnancy enhanced in infertile patients who used IUI, which is of great importance due to its lower costs. Additionally, there was no relationship between the infertility type and the abortion or pregnancy rates of the couples. Besides, no significant relationship was observed between pregnancy rate and BMI, the duration of infertility or dominant follicles. However, it is strongly recommended that this study be conducted with a larger sample size and a reduced number of couples with unknown etiology of infertility.

\section{Conflict of Interests}

Authors declare that they have no conflict of interests.

\section{Ethical Issues}

This study was approved by the Ethics Committee of 
Tabriz University of Medical Sciences. All patients were given informed consent. Additionally, this study was registered in Iranian Registry of Clinical Trials (identifier: IRCT2016110213566N7; https://www.irct.ir/trial/13385). The aims of the study, the intervention and the reason why they were chosen for the study were explained to all the patients and they all filled an informed consent form. Though the treatments in this study were the routine treatments, possible side-effects of the treatment and downsides of the study were explained. We ensured that their data would remain confidential. All procedures performed in this study were in accordance with the ethical standards of the institutional and national research committee and with the 1964 Declaration Helsinki.

\section{Financial Support}

This article had no financial support.

\section{References}

1. Rowe PJ, Comhaire FH. WHO manual for the standardized investigation and diagnosis of the infertile male. Cambridge University Press; 2000.

2. Cooper TG, Noonan E, von Eckardstein S, et al. World Health Organization reference values for human semen characteristics. Hum Reprod Update. 2010;16(3):231-245. doi:10.1093/humupd/dmp048

3. Parsanezhad ME, Dadras N, Maharlouei N, Neghahban L, Keramati P, Amini M. Pregnancy rate after endometrial injury in couples with unexplained infertility: A randomized clinical trial. Iran J Reprod Med. 2013;11(11):869-874.

4. Effectiveness and treatment for unexplained infertility. Fertil Steril. 2006;86(5 Suppl 1):S111-114. doi:10.1016/j. fertnstert.2006.07.1475

5. Margalioth EJ, Ben-Chetrit A, Gal M, Eldar-Geva T. Investigation and treatment of repeated implantation failure following IVF-ET. Hum Reprod. 2006;21(12):30363043. doi:10.1093/humrep/del305

6. Simon C, Bellver J. Scratching beneath 'The Scratching
Case': systematic reviews and meta-analyses, the back door for evidence-based medicine. Hum Reprod. 2014;29(8):1618-1621. doi:10.1093/humrep/deu126

7. Barash A, Dekel N, Fieldust S, Segal I, Schechtman E, Granot I. Local injury to the endometrium doubles the incidence of successful pregnancies in patients undergoing in vitro fertilization. Fertil Steril. 2003;79(6):1317-1322.

8. Almog B, Shalom-Paz E, Dufort D, Tulandi T. Promoting implantation by local injury to the endometrium. Fertil Steril. 2010;94(6):2026-2029. doi:10.1016/j. fertnstert.2009.12.075

9. Zhou L, Li R, Wang R, Huang HX, Zhong K. Local injury to the endometrium in controlled ovarian hyperstimulation cycles improves implantation rates. Fertil Steril. 2008;89(5):1166-1176. doi:10.1016/j.fertnstert.2007.05.064

10. Zarei A, Alborzi S, Dadras N, Azadi G. The effects of endometrial injury on intrauterine insemination outcome: A randomized clinical trial. Iran J Reprod Med. 2014;12(9):649-652.

11. Karimzade MA, Oskouian H, Ahmadi S, Oskouian L. Local injury to the endometrium on the day of oocyte retrieval has a negative impact on implantation in assisted reproductive cycles: a randomized controlled trial. Arch Gynecol Obstet. 2010;281(3):499-503. doi:10.1007/s00404-009-1166-1

12. Intrauterine insemination. Hum Reprod Update. 2009;15(3):265-277. doi:10.1093/humupd/dmp003

13. Jasovic V, Jasovic-Siveska E. [Success rate of intrauterine insemination in patients with unknown infertility]. Vojnosanit Pregl. 2012;69(4):301-307.

14. Maged AM, Al-Inany H, Salama KM, Souidan, II, Abo Ragab HM, Elnassery N. Endometrial scratch injury induces higher pregnancy rate for women with unexplained infertility undergoing IUI with ovarian stimulation: a randomized controlled trial. Reprod Sci. 2016;23(2):239243. doi:10.1177/1933719115602776

15. Abdelhamid AM. The success rate of pregnancy in IUI cycles following endometrial sampling. A randomized controlled study: endometrial sampling and pregnancy rates. Arch Gynecol Obstet. 2013;288(3):673-678. doi:10.1007/s00404013-2785-0

(c) 2019 The Author (s); This is an open-access article distributed under the terms of the Creative Commons Attribution License (http://creativecommons.org/licenses/by/4.0), which permits unrestricted use, distribution, and reproduction in any medium, provided the original work is properly cited. 